\title{
Interface and Requirements Analysis on the DEMO Heating and Current Drive System Using Systems Engineering Methodologies
}

\author{
${ }^{\mathrm{a}}$ Giovanni Grossetti, ${ }^{\mathrm{b}}$ Christophe Baylard, ${ }^{\mathrm{b}, \mathrm{d}}$ Thomas Franke, ${ }^{\mathrm{a} J u ̈ r g e n ~ G a f e r t, ~}{ }^{\mathrm{c}}$ Ian Jenkins, \\ ${ }^{b, d}$ Mattia Siccinio, ${ }^{a}$ Dirk Strauß, ${ }^{\mathrm{e}}$ Minh Quang Tran, ${ }^{\mathrm{d}}$ Hartmut Zohm \\ ${ }^{a}$ Karlsruhe Institute of Technology, P.O. Box 3640, D-76021 Karlsruhe, Germany \\ ${ }^{b}$ EUROfusion Programme Management Unit, Boltzmannstr. 2, D-85748 Garching, Germany \\ ${ }^{c}$ Culham Centre for Fusion Energy, Abingdon, OX14 3DB, Oxfordshire, U.K. \\ ${ }^{d}$ Max-Planck-Institut für Plasmaphysik, Boltzmannstr. 2, D-85748 Garching, Germany \\ ${ }^{e}$ Swiss Plasma Center EPFL, CH-1015 Lausanne, Switzerland
}

*Corresponding author: giovanni.grossetti@kit.edu

In this paper we present the methodology implemented for analyzing System Requirements and Interfaces of the Heating and Current Drive (HCD) system of the European Demonstration Fusion Power Reactor DEMO.

The work consisted in updating the preliminary framework of the Model-Based Systems Engineering model of the HCD System Architecture. This is now containing an ontology, a set of 6 perspectives and a defined set of viewpoints for each Perspective, for refining the HCD System Architecture.

The scope of the work is to manage the interdependencies of HCD system elements and their integration into DEMO, for a given set of system functions. On the one hand, this means to address the identification and definition of the interfaces occurring, both internally in the HCD system, and between the HCD system and neighboring systems. On the other hand, this implies studying the impact of requirements coming from the ongoing physics studies.

The rationale is to provide the technical foreground for supporting the decision-making processes related to the HCD system which is planned to be carried out during the forthcoming Conceptual Design Phase.

The results we show in this paper are part of the design and integration activities consisting of both systems engineering methodologies and design analysis, all aiming at ensuring consistency in the overall EU DEMO plant design. In this framework the DEMO Heating and Current Drive system has been selected as pilot project for the application of Systems Engineering methodologies.

Keywords: Nuclear Fusion, DEMO, Heating and Current Drive, Systems Engineering, MBSE.

\section{Introduction}

The European Consortium for the development of fusion energy - EUROfusion [1] and in particular its Power Plant Physics and Technology department (PPPT) is undertaking R\&D activities for the DEMOnstration Power plant DEMO [2-4]. The project is in PreConceptual Design Phase in which different technologies and plasma scenarios are under assessment. In this frame, the Work Package Heating and Current Drive (WPHCD) is in charge of developing the concepts and the technologies for a reliable and efficient Heating and Current Drive (HCD) system [5]. The purpose of the HCD system is to provide the appropriate power and/or to generate current into the plasma such that fusion reactions can occur. Presently, three different heating methods are considered, i.e. Electron Cyclotron Heating (ECH) [6-7], Ion Cyclotron Heating (ICH) [8] and Neutral Beam Injection (NBI) [9-10]. The HCD system must be fully integrated into DEMO, for which two main concepts are currently considered: i) a DEMO 1 concept based on the expected performances of ITER with an improved technology; ii) an "optimistic design" (FlexiDEMO), capable to be operated as steady-state. In PPPT for supporting system design tasks, the Work Package
Plant Level System Engineering, Design Integration and Physics Integration (WPPMI) systematically follows a practical approach consisting of a set of Model-Based System Engineering (MBSE) processes. The scope of this iterative work is to tailor System Engineering methodologies in order to support the design and integration of the HCD system in DEMO. Besides proper requirement engineering activities that grants traceability and monitoring of requirements, we initiated the development of a system model using the SysML language for supporting the identification of both external and internal interfaces, the definition of the different system elements, and the systematic analysis of requirement. Due to the current phase, the task aims also to support, technology and design options evaluation.

The work started in 2017 [11], focusing on identifying the stakeholder needs and in capturing initial system requirements and functions. In this paper we bring forward that previous analysis, using a framework develop by CCFE.

The paper is divided as follows: in section 2 we will introduce MBSE and we will briefly explain the framework used; in section 3 we will discuss the perspectives and views applied to the HCD system, showing a few examples of use cases studies and 
structures; in section 4 we will provide the conclusions and an outlook for future work.

\section{The importance of Systems Engineering in the early design phase}

The development of the HCD system is challenged by different issues, such as: Stakeholder requirements only preliminary elaborated, uncertainties in physics assumptions and clear targets of the machine not been yet confirmed, further assessments on materials for plasma-facing components [12], and distance between research groups distributed over the whole European Union. Therefore, a methodology focusing on the design and application of the whole system, looking at it in its entirety and taking into account the social and technical aspect, can strongly support the development, mitigating the risks associated to floating requirements and targets. In the next paragraph, we will provide a brief overview of the approach we implemented in this work.

\subsection{MBSE methodologies: System Architecture and its Framework, and requirements identification}

Following INCOSE (International Consortium for System Engineering) definition [13], MBSE is the formalized application of modelling to support system requirements, design, analysis, verification and validation activities beginning in the conceptual design phase and continuing throughout development and later life cycle phases. Using MBSE and a proper modelling language, one can develop a representation of a System Of Interest (SOI) consisting in a series of views or diagrams aiming to describe the SOI itself under a number of different perspectives. Such a representation is called System Architecture. For the DEMO project the language used is the System Modelling Language (SysML) [14]. The work we present here is part of integration activities, consisting of both systems engineering methodologies compliant with the standard ISO/IEC/IEEE 15288 [15]. The preliminary HCD system architecture developed in 2017 and presented in [11] has been updated using a framework, i.e. a set of rules to establish a common practice for creating, interpreting, analyzing and using architecture descriptions within a particular domain of application or stakeholder community (ISO/IEC/IEEE 1471:2007), called DEMO AF (DEMO Architectural Framework). This is now containing an ontology, a set of six perspectives (namely System, Needs, Traceability, Maintenance, Deployment and Operational) and a defined set of viewpoints for each Perspective, where each Viewpoint is a template for a set of Views. In the work currently done, we have analyzed booth the System and Need Perspectives. These are the two perspectives with which, in general, any architecture work starts. The system perspective presents what the system is (also called "physical architecture") and the needs perspectives presents what the system does (its functions and constraints), often called the logical architecture.
Any SOI is defined by a suitable set of requirements that feed the technical specification for the design development phase. Being DEMO a first-of-its-kind machine, the derivation for its requirements and their propagation to its subsystems, is a non-trivial effort. The approach we are currently following for the derivation of the requirements for the HCD system is the typical TopDown approach (see [11]). Important to note is that despite the several ongoing design assessments on possibly interfacing systems, interfaces and interface requirements have been not fully yet explored.

We would also like to remark that due to space limitation, we report only a few examples of the views developed, representing therefore an extract of the entire system model that includes views for EC, IC and NB.

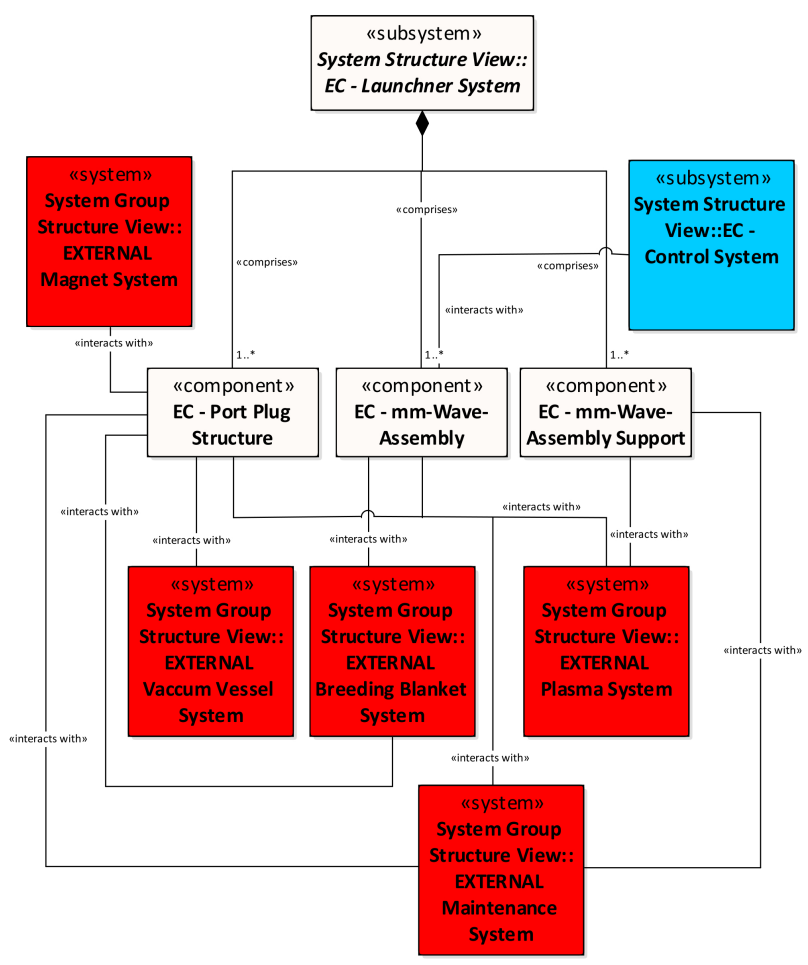

Fig. 1. Example of a System Structure View representing the EC Launcher system. The red boxes represent external (to the EC system) interfaces; the blue box represents internal interfaces.

\section{HCD Analysis: System Perspectives}

The physical layer of the System Architecture is represented by the System Perspectives. These are used to provide an understanding of the system and to define the interfaces between the various components. It lists the physical attributes of a SOI and provides a description of the relationships between the different system elements following a structure established in the Product Breakdown Structure (PBS) developed for the DEMO project. The views contained in the system perspectives are:

i. the System Structure Views, that provides a graphical decomposition of a SOI in its system elements; the relationships between system elements are generally indicating a hierarchy or an ownership. 
Figure 1 describes the Structure View for the subsystem 'EC Launcher': The different boxes represent different system elements that area part of the Launcher system (white), internal interfaces (blue) or external interfaces (red).

ii. A System Configuration View, illustrating the relationships between system elements by connecting them through specific Ports and Flow Types. In figure 2 is shown an example for such a view for the NB system. It is important to note that for graphical reasons only a part of the Configuration View is shown (the complete view is too complex for being properly represented on such a publication). Also, the dimensions of the rectangles representing the system elements have no physical meaning, but it has been defined according to graphical needs.

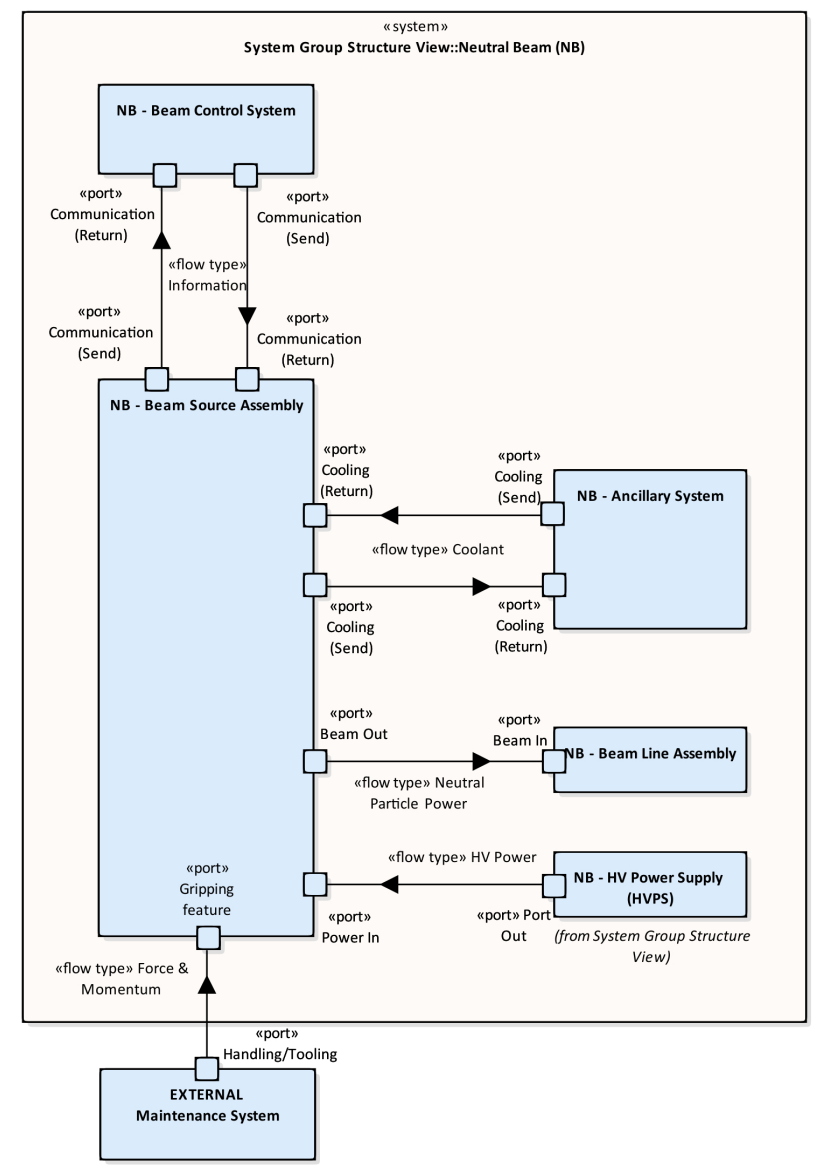

Fig. 2. Example of a system configuration view for the NB system, including port types and flow types. Note that also for NB - Beam Control Systems, Ancillary System, Beam Line Assembly and HVPS are foreseen inlet and outlet flows, not reported in the figure for readability purposes.

Here it is important to remark that for each, subsystem, internal/external interface and function a proper set of requirements will be developed in the following years, since the requirements will drive the design of the system and its integration with the neighbouring ones. On each system elements are placed ports. In the example of figure 2 , these can be communication ports associated to control signals, cooling ports interfacing with cooling manifolds, beam ports related to neutral particles input and output, gripping feature and handling/tooling ports related to maintenance aspects and power ports identifying the interfaces with high voltage systems. Connections between ports define the nature of the flow occurring between them. In the example of figure 2 we have considered information, coolant, particle power and, force and momentum.

\section{HCD Analysis: Needs Perspectives}

The Logical layer of the System Architecture is represented by the Needs Perspectives. These provide the goals for the HCD system within a specific context or viewpoint. The goals are called Use Cases and they shall be associated to System Requirements. This kind of analysis are indispensable for complex for two main reasons: i) to define in a systematic and traceable way the needs and goals of the SOI, by defining the functions associated to the system elements of the SOI; ii) support in a next step the requirement drafting by highlighting "missing" requirements, i.e. requirements not yet captured although the specific Use Case is being defined.

In figure 3 it is shown a typical Use Case study develop for the HCD system. This consist of:

i. a boundary (black solid rectangle) representing the context with which the analysis is being framed;

ii. a set of balloons representing the goals (Use Cases) which shall be associated to specific sets of requirements.

iii. Relationships among Use Cases are represented by dashed lines. In the example of figure 3 , these can be "include" or "constrain". Outside the boundary, a number of stakeholders for the HCD system in this context are shown. Four of them are associated with Work Packages, i.e. DEMO sub-projects, having interfaces with the HCD system (for this reason they are all associated to the Use Case "Define System Boundaries and Interfaces"). More specifically, these are the Work Packages Breeding Blanket (WPBB), Diagnostic and Control (WPDC), Magnets (WPMAG) and Remote Maintenance (WPRM). A fifth stakeholder representing the PMU Lead System Integrator - LSI (the DEMO responsible Officer) is also included for port allocation and integration with confinement barriers. It is important to note that the list of stakeholders is not exhaustive, but considers only the once for which the interfaces have been at least preliminary defined.

Also for figure 3, it must be noted that what shown represents a portion of the entire view, since the complete Use Case Study was too large for being completely represented here. The Use Case "Defining system boundaries and interfaces" represents a major driver for the HCD System development and it involves a number of stakeholders. In the forthcoming years focus will be given to trace this Use Case by a suitable set of requirements to be defined in cooperation with stakeholders. The needs perspectives offer a unique tool for understanding how the different goals and functions of a SOI are related to one another. Considering the HCD system, we are still at the beginning of the 
development. Further refinement and updates are necessary before having the complete description of the HCD system from the functional point of view.

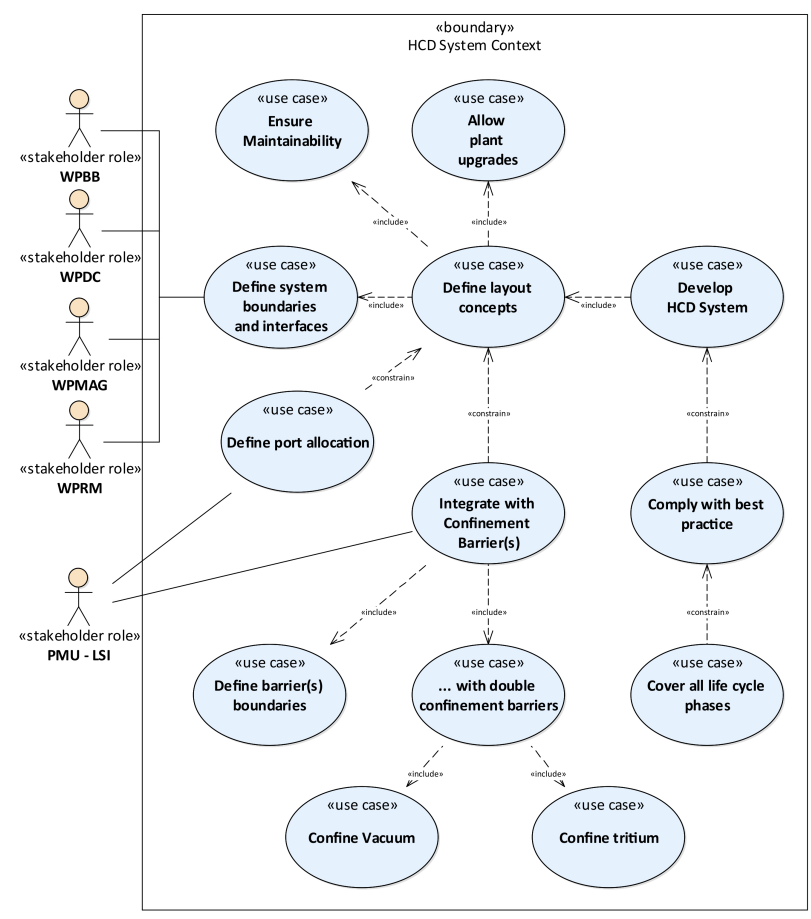

Fig. 3. Use Case Diagram for the HCD System Context, showing the Use Cases and the Actors (interfacing system elements or entities) relevant in this early development phase.

\section{Conclusions}

Following the prioritization by PMU on the use of System Engineering methodologies (where the HCD system is used as pilot project) in such a large and longterm project as DEMO, we have presented the update of the HCD System Architecture. This consisted in the application of MBSE methodologies with the aim of a) developing the HCD System(s) Architecture and b) to define a systematic approach to record and assess the impact of requirement evolution. The work has been done addressing the Logical and Physical layers of the System Architecture, by assessing structures and needs perspectives. The introduction of the DEMO Framework helped considerably in defining a number of consistent views dealing with both physical as well as logical attributes. Strong cooperation between WPHCD, WPPMI and the systems engineers at KIT and CCFE allowed a sensible leap forward in the architecture development. The next steps currently under assessments will consist in refining the functional requirements following the latest results from performance analyses which will lead to an updated of needs perspectives, including quantitative input from physics assessments.

\section{Acknowledgments}

This work has been carried out within the framework of the EUROfusion Consortium and has received funding from the EURATOM research and training programme
2014-2018 under grant agreement No 633053. The views and opinions expressed herein do not necessarily reflect those of the European Commission.

\section{References}

[1] T. Donnè, W. Morris, "European Research Roadmap to the realization of Fusion Energy" EUROfusion pub. 2018, www.eurofusion .org/eurofusion/roadmap

[2] G. Federici et al., "DEMO Design Activity in Europe: Progress and Updates", Volume 136, Part A November 2018, Pages 729-741

[3] M. Coleman et al., "On the EU approach for DEMO architecture exploration and dealing uncertainties", Fus. Eng. \& Des. 109-111 (2016) 1158-1162

[4] F. Maviglia et al., Optimization of DEMO geometry and disruption location prediction, In Press on Fus. Eng \& Des. (2019)

[5] M. Q. Tran et al. ,R\&D in the frame of the PPPT Heating and Current Drive for EU DEMO“, $22^{\text {nd }}$ Radiofrequency Power in Plasma Topical Conference (RFPPC), May $30^{\text {th }}-$ June $2^{\text {nd }}, 2017$, Aix en Provence (France)

[6] S. Garavaglia et al., "EU DEMO EC system preliminary conceptual design”, Volume 136, Part B, November 2018, Pages 1173-1177

[7] G. Grossetti et al., "DEMO port plug design and integration studies" 2017 Nucl. Fusion 57116028

[8] A. Bader et al., "Integrating a distributed antenna DEMO: requirements and challenges“, Volume 123, November 2017, Pages 431-434

[9] P. Sonato et al., "Conceptual design of the DEMO neutral beam injectors: main developments and R\&D achievements", Nucl. Fusion 57 (2017) 056026

[10] C. Hopf et al., "A conceptual system design study for an NBI beamline”, In Press on Fus. Eng \& Des. (2019)

[11] G. Grossetti, et al., "Systems engineering perspective to the integration of the heating and current drive system in the EU DEMO: Analysis of requirements and functions", Fus. Eng. \& Des. 136 (2018) 53-57

[12] Aljaž Čufar et al., Equatorial electron cyclotron port plug neutronic analyses for the EU DEMO, In Press on Fus. Eng \& Des. (2019)

[13] INCOSE. 2007. Systems Engineering Vision 2020. INCOSE-TP-2004-004-02 September, 2007.

[14] P. Pearce et al., "A practical approach for modelling submarine subsystem architecture in SysML", Submarine Institute of Australia Science, Technology \& Engineering Conference 2013

[15] ISO/IEC/IEEE. 2015. Systems and Software Engineering - System Life Cycle Processes. Geneva, Switzerland: International Organization for Standardization (ISO)/International Electrotechnical Commission (IEC)/Institute of Electrical and Electronics Engineers (IEEE). ISO/IEC/IEEE 15288:2015 (E). 